\title{
THE SPACE OF RETRACTIONS OF A COMPACT $Q$-MANIFOLD IS AN $l^{2}$-MANIFOLD
}

\author{
KATSURO SAKAI
}

\begin{abstract}
In this paper, we prove that the space of retractions of a compact Hilbert cube manifold is an $l^{2}$-manifold. This answers a question raised by $\mathrm{T}$. $A$. Chapman.
\end{abstract}

Let $M$ be a compact $Q$-manifold, and let $R(M)$ be the space of retractions of $M$, equipped with the sup-metric, i.e., $R(M)=\left\{e \mid e: M \rightarrow M\right.$ is continuous, $\left.e^{2}=e\right\}$. T. A. Chapman [2] proved that $R(M)$ is an ANR, and he asked whether $R(M)$ is an $l^{2}$-manifold. The purpose of this paper is to answer this question affirmatively.

TheOREM. $R(M)$ is an $l^{2}$-manifold.

Recently, H. Toruńczyk gave the mapping characterization of $l^{2}$-manifolds [3, Corollary 3.3], which states that a separable complete-metrizable ANR $X$ is an $l^{2}$-manifold if and only if the following two conditions are satisfied:

(*) For each $n \in \mathrm{N}$, any two continuous maps $f, g: I^{n} \rightarrow X$ can be arbitrarily closely approximated by continuous maps with disjoint images.

(**) For any sequence $\left\{P_{n}\right\}_{n \in N}$ of compact polyhedra, any continuous map $f$ : $\sum_{n \in \mathrm{N}} P_{n} \rightarrow X$ can be arbitrarily closely approximated by a continuous map $g$ : $\sum_{n \in \mathrm{N}} P_{n} \rightarrow X$ such that $\left\{g\left(P_{n}\right)\right\}_{n \in \mathrm{N}}$ is locally finite in $X$.

Actually, the condition (*) is unnecessary, ${ }^{1}$ that is, the condition $(* *)$ implies the condition (*) since, as noted in [4], if $g_{i}: I^{n} \rightarrow X(i \in \mathbf{N})$ are approximations of a continuous map $g: I^{n} \rightarrow X$ such that $\left\{g_{i}\left(I^{n}\right)\right\}_{i \in \mathrm{N}}$ is locally finite in $X$, then for every compact subset $K$ of $X, g_{i}\left(I^{n}\right)$ is disjoint from $K$ for almost all $i \in \mathbf{N}$. Thus, it suffices to show that $R(M)$ satisfies the condition (**).

Since $M$ is homeomorphic to $M \times Q$ [1], we may show that $R(M \times Q)$ satisfies the condition (**). Points of $M \times Q$ will be denoted by $y=\left(y_{0}, y_{1}, y_{2}, \ldots\right)$, where $y_{0} \in M$ and $y_{i} \in I_{i}=[-1,1] \quad(i=1,2, \ldots)$. We use the metric on $M \times Q$ defined by

$$
d\left(y, y^{\prime}\right)=d_{M}\left(y_{0}, y_{0}^{\prime}\right)+\sum_{i=1}^{\infty} 2^{-i}\left|y_{i}-y_{i}^{\prime}\right|,
$$

where $d_{M}$ is a metric on $M . R(M \times Q)$ is equipped with the sup-metric $d\left(e, e^{\prime}\right)=$ $\sup \left\{d\left(e(y), e^{\prime}(y)\right) \mid y \in M \times Q\right\}$.

Received by the editors July 22, 1980 and, in revised form, February 12, 1981; presented to the Society of Japan, October 1, 1980.

1980 Mathematics Subject Classification. Primary 54C35, 58D15, 57N20.

$K e y$ words and phrases. Space of retractions, ANR, $Q$-manifold, $l^{2}$-manifold.

${ }^{1} D$. W. Curtis suggested to me that $J$. van Mill noted this. 
An ambient invertible isotopy $h_{t}(t \in[1, \infty))$. We will define an ambient invertible isotopy $h_{t}:(M \times Q) \times Q \rightarrow M \times Q(t \in[1, \infty))$. First we define homeomorphisms $h_{i}, h_{i+j / i}:(M \times Q) \times Q \rightarrow M \times Q(i=1,2, \ldots ; j=1, \ldots, i-1)$ as follows.

$$
\begin{aligned}
& h_{1}(y, z)=\left(y_{0}, y_{1}, z_{1}, y_{2}, z_{2}, y_{3}, z_{3}, y_{4}, z_{4}, \ldots\right) \text {, } \\
& h_{2}(y, z)=\left(y_{0}, y_{1}, y_{2},-z_{1}, z_{2}, y_{3}, z_{3}, y_{4}, z_{4}, \ldots\right) \text {, } \\
& h_{2+(1 / 2)}(y, z)=\left(y_{0}, y_{1}, y_{2},-z_{1}, y_{3},-z_{2}, z_{3}, y_{4}, z_{4} \ldots\right) \text {, } \\
& h_{3}(y, z)=\left(y_{0}, y_{1}, y_{2}, y_{3}, z_{1},-z_{2}, z_{3}, y_{4}, z_{4}, \ldots\right) \text {, } \\
& h_{3+(1 / 3)}(y, z)=\left(y_{0}, y_{1}, y_{2}, y_{3}, z_{1},-z_{2}, y_{4},-z_{3}, z_{4} \ldots\right) \text {, } \\
& h_{3+(2 / 3)}(y, z)=\left(y_{0}, y_{1}, y_{2}, y_{3}, z_{1}, y_{4}, z_{2},-z_{3}, z_{4}, \ldots\right) \text {, } \\
& h_{4}(y, z)=\left(y_{0}, y_{1}, y_{2}, y_{3}, y_{4},-z_{1}, z_{2},-z_{3}, z_{4}, \ldots\right) \text {, } \\
& h_{i}(y, z)=\left(y_{0}, \ldots, y_{i},(-1)^{i-1} z_{1},(-1)^{i-2} z_{2}, \ldots,(-1) z_{i-1}, z_{i}\right. \text {, } \\
& \left.y_{i+1}, z_{i+1}, y_{i+2}, z_{i+2}, \ldots\right) \text {, } \\
& h_{i+(j / i)}(y, z)=\left(y_{0}, \ldots, y_{i},(-1)^{i-1} z_{1}, \ldots,(-1)^{j} z_{i-j}, y_{i+1}\right. \text {, } \\
& \left.(-1)^{j} z_{i-j+1}, \ldots,(-1) z_{i}, z_{i+1}, y_{i+2}, z_{i+2}, \ldots\right) \text {, } \\
& h_{i+1}(y, z)=\left(y_{0}, \ldots, y_{i+1},(-1)^{(i+1)-1} z_{1}, \ldots,(-1) z_{i}, z_{i+1}\right. \text {, } \\
& \left.y_{i+2}, z_{i+2}, y_{i+3}, z_{i+3}, \ldots\right)
\end{aligned}
$$

Let $\theta_{t}:[-1,1]^{2} \rightarrow[-1,1]^{2}(t \in I)$, be an ambient invertible isotopy such that $\theta_{0}=$ id, $\theta_{1}\left(s_{1}, s_{2}\right)=\left(s_{2},-s_{1}\right)$ for each $\left(s_{1}, s_{2}\right) \in[-1,1]^{2}$. For each

$$
t \in[i+(j-1) / i, i+j / i] \quad(i=1,2,3, \ldots ; j=1, \ldots, i)
$$

we define

$$
h_{t}=\theta_{i(t-i-(j-1) / i)}^{i, j} \circ h_{i+(j-1) / i}:(M \times Q) \times Q \rightarrow M \times Q,
$$

where $\theta_{t}^{i, j}: M \times Q \rightarrow M \times Q(t \in I)$ is an ambient invertible isotopy defined by

$$
\theta_{t}^{i, j}(y)=\left(y_{0}, \ldots, y_{2 i-j}, \theta_{t}\left(y_{2 i-j+1}, y_{2 i-j+2}\right), y_{2 i-j+3}, \ldots\right) \text {. }
$$

Note that our ambient invertible isotopy $h_{t}(t \in[1, \infty))$ has the following properties:

(1) If $t<i$, then $p_{2 i} h_{t}(y, z)=z_{i}$ for all $(y, z) \in(M \times Q) \times Q$,

(2) If $t>i$, then $p_{i} h_{t}(y, z)=y_{i}$ for all $(y, z) \in(M \times Q) \times Q$. 
ProOF OF THE CONDITION (**). Let $\left\{P_{n}\right\}_{n \in N}$ be a sequence of compact polyhedra and $f: \Sigma_{n \in \mathrm{N}} P_{n} \rightarrow R(M \times Q)$ a continuous map. For any continuous function $\varepsilon$ : $R(M \times Q) \rightarrow(0, \infty)$, there exists a continuous function $\delta: r(M \times Q) \rightarrow(0,1]$ such that $\delta(e) \leqslant \varepsilon(e) / 2$ and $d\left(y, y^{\prime}\right)<\delta(e)\left(y, y^{\prime} \in M \times Q\right)$ implies $d\left(e(y), e\left(y^{\prime}\right)\right)$ $<\varepsilon(e) / 2$ for each $e \in R(M \times Q)$. (This is because the function $\bar{\delta}: R(M \times Q) \rightarrow$ $(0, \infty)$ defined by $\bar{\delta}(e)=\sup \left\{\delta>0 \mid d\left(y, y^{\prime}\right)<\delta \Rightarrow d\left(e(y), e\left(y^{\prime}\right)\right)<\varepsilon(e) / 2\right\}$ is lower semicontinuous.)

Each $P_{n}$ admits a triangulation $K_{n}$ such that

$$
\sup \{\delta f(x) \mid x \in \sigma\}-\inf \{\delta f(x) \mid x \in \sigma\}<2^{-n}
$$

and

$$
\sup \{\delta f(x) \mid x \in \sigma\}<2 \inf \{\delta f(x) \mid x \in \sigma\}
$$

for each simplex $\sigma$ of $K_{n}$. For each $x \in P_{n}$, let $(x(v))_{v \in K_{n}^{0}}$ be the barycentric coordinates of $x$ with respect to the triangulation $K_{n}$. For each vertex $v$ of $K_{n}$, choose a positive integer $i(v)$ so that $2^{-i(v)+2}<\delta f(v)<2^{-i(v)+3}$ and define a continuous function $t: \sum_{n \in \mathrm{N}} P_{n} \rightarrow[1, \infty)$ by

$$
t(x)=\sum_{v \in K_{n}^{0}} x(v) i(v) \text { for each } x \in P_{n} .
$$

For each $n \in \mathbf{N}$, let $r_{n}:[-1,1] \rightarrow[-1,1]$ be a piecewise-linear map such that $r_{n}(-1)=r_{n}(0)=r_{n}(1)=1$ and $r_{n}(1 / n)=1 / n$. Then define $r_{n}^{*}: Q \rightarrow Q$ by

$$
r_{n}^{*}\left(z_{1}, z_{2}, \ldots\right)=\left(r_{n}\left(z_{1}\right), r_{n}\left(z_{2}\right), \ldots\right) \text {. }
$$

Now, we define a map $g: \Sigma_{n \in \mathrm{N}} P_{n} \rightarrow R(M \times Q)$ by

$$
g(x)=h_{t(x)} \circ\left(f(x) \times r_{n}^{*}\right) \circ h_{t(x)}^{-1} \quad \text { for each } x \in P_{n} .
$$

Since $h_{t}(t \in[1, \infty))$ is an ambient invertible isotopy, $g$ is continuous. We assert that $g$ is a desired approximation of $f$.

First, we see that $d(f(x), g(x))<\varepsilon f(x)$ for each $x \in \Sigma_{n \in N} P_{n}$. Let $x \in \sigma \in K_{n}$. There exists a vertex $v$ of $\sigma$ such that $i(v) \leqslant t(x)$. From (2) and (4), $d\left(h_{t(x)}, p\right)<$ $2^{-i(v)+1} \leqslant \delta f(v) / 2<\delta f(x)$ where $p:(M \times Q) \times Q \rightarrow M \times Q$ is the projection. Hence

$$
d\left(f(x) p, f(x) h_{t(x)}\right)<\varepsilon f(x) / 2
$$

Thus

$$
\begin{aligned}
d(f(x), g(x)) & \leqslant d\left(f(x), p\left(f(x) \times r_{n}^{*}\right) h_{t(x)}^{-1}\right)+d\left(p\left(f(x) \times r_{n}^{*}\right) h_{t(x)}^{-1}, g(x)\right) \\
& =d\left(f(x) h_{t(x)}^{-1}, p\left(f(x) \times r_{n}^{*}\right)\right)+d\left(p, h_{t(x)}\right) \\
& <d\left(f(x) h_{t(x)}^{-1}, f(x) p\right)+\delta f(x)<\varepsilon f(x) .
\end{aligned}
$$

Next, we claim that $\left\{g\left(P_{n}\right)\right\}_{n \in N}$ is locally finite in $R(M \times Q)$. Suppose not. Then there exists a convergent sequence $g\left(x_{n_{i}}\right) \rightarrow e \in R(M \times Q)$, where $x_{n_{1}} \in P_{n_{1}}$ for each $i \in \mathrm{N}$. For convenience, assume $n_{i}=n$; thus $g\left(x_{n}\right) \rightarrow e(n \rightarrow \infty), x_{n} \in P_{n}$. If there exists a positive integer $i_{0}$ such that $t\left(x_{n}\right)<i_{0}$ for each $n \in \mathbf{N}$, then $p_{2 i_{0}} g\left(x_{n}\right)=r_{n} p_{2 i_{0}}$ from (1), where $p_{i}: M \times Q \rightarrow I_{i}$ is the projection. This is a 
contradiction, because $p_{2 i_{0}} g\left(x_{n}\right) \rightarrow p_{2 i_{0}} e$ but $r_{n} p_{2 i_{0}}$ cannot converge to any continuous function. Thus $\left\{t\left(x_{n}\right)\right\}_{n \in N}$ is unbounded. Hence we may assume that $t\left(x_{n}\right) \rightarrow$ $\infty \quad(n \rightarrow \infty)$. Then $h_{t\left(x_{n}\right)} \rightarrow p$, so $d\left(g\left(x_{n}\right), p\left(f\left(x_{n}\right) \times r_{n}^{*}\right) h_{t\left(x_{n}\right)}^{-1}\right) \rightarrow 0$. Hence $p\left(f\left(x_{n}\right) \times r_{n}^{*}\right) h_{t\left(x_{n}\right)}^{-1} \rightarrow e$, so $d\left(p\left(f\left(x_{n}\right) \times r_{n}^{*}\right), e h_{t\left(x_{n}\right)}^{-1}\right) \rightarrow 0$. Since $e h_{t\left(x_{n}\right)} \rightarrow e p, f\left(x_{n}\right) p=$ $p\left(f\left(x_{n}\right) \times r_{n}^{*}\right) \rightarrow e p$. Therefore $f\left(x_{n}\right) \rightarrow e$ because $p$ is onto. On the other hand, there are vertices $v_{n}$ of the carriers of $x_{n}$ such that $t\left(x_{n}\right)<i\left(v_{n}\right)$. Since $\delta f\left(v_{n}\right)<$ $2^{-i\left(v_{n}\right)+3}$ and $t\left(x_{n}\right) \rightarrow \infty, \delta f\left(v_{n}\right) \rightarrow 0$. From (3), $\left|\delta f\left(v_{n}\right)-\delta f\left(x_{n}\right)\right|<2^{-n}$, then $\delta f\left(x_{n}\right) \rightarrow$ 0 . Hence $\delta(e)=0$. This is a contradiction.

I would like to express my thanks to D. W. Curtis for helpful suggestions which simplify my arguments in the proof.

\section{REFERENCES}

1. R. D. Anderson and R. M. Schori, Factors of infinite-dimensional manifolds, Trans. Amer. Math. Soc. 142 (1969), 315-330.

2. T. A. Chapman, The space of retractions of a compact Hilbert cube manifold is an ANR, Topology Proc. 2 (1977), 409-430.

3. H. Torunczyk, Characterizing Hilbert space topology, Inst. Math. Polish Acad. Sci., preprint 143.

4. R. D. Anderson, D. W. Curtis and J. van Mill, A fake topological Hilbert space, Trans. Amer. Math. Soc. (to appear).

INSTITUTE OF MATHEMATICS, UNIVERSITY OF TSUKUBA, IBARAKI, JAPAN 